\title{
CONTROLE DE ROBÔS MANIPULADORES EM MODO DUAL ADAPTATIVO/ROBUSTO
}

\author{
Fernando A. Pazos* \\ quinieort.org.br
}

\author{
Liu Hsu* \\ liu@coep.ufrj.br
}

* Programa de Engenharia Elétrica - COPPE/UFRJ, C.P. 68504, 21945/970 - Rio de Janeiro, RJ, BRASIL

\begin{abstract}
We consider the trajectory tracking problem for robot manipulators with unknown physical parameters and affected by bounded external disturbances. We present a non linear adaptive tracking controller by dual mode which guarantees exponential transient performance with respect to an arbitrary small residual set, resulting in arbitrary disturbance attenuation. If the disturbances vanish the controller achieves asymptotic tracking of the desired trajectory.
\end{abstract}

KEYWORDS: Adaptive/robust control, disturbance rejection, exponential tracking trajectory.

\section{RESUMO}

Considera-se o problema de rastreamento de trajetórias para robôs manipuladores com parâmetros físicos incertos e sob a ação de distúrbios de amplitude limitada. Descreve-se um algoritmo de controle de modo dual adaptativo/robusto que garante desempenho transitório exponencial com relação a um domínio residual arbitrariamente pequeno e com atenuação arbitrária de distúrbios. Na ausência destes, o controlador mostra-se capaz de rastrear assintoticamente a trajetória desejada.

PALAVRAS-CHAVE: Controle adaptativo/robusto, atenuação de distúrbios, rastreamento de trajetória exponencial.

\footnotetext{
Artigo submetido em 04/12/00

1a. Revisão em 10/04/02

Aceito sob recomendação do Ed. Assoc. Prof. Paulo E. Miyagi
}

\section{INTRODUÇÃO}

Atualmente, já são bem conhecidas diversas leis de controle adaptativo de manipuladores robóticos baseadas em identificação paramétrica (Bayard e Wen, 1988; Craig, 1986). Entretanto, a qualidade do desempenho transitório de adaptação não foi caracterizada e a garantia de robustez com relação a distúrbios e outras imperfeições do sistema requer modificações das leis de adaptação, e, mesmo assim, a robustez é geralmente garantida só em termos da limitação dos sinais do sistema, o que na prática é insuficiente.

Um objetivo mais satisfatório seria conseguir uma lei de controle que resultasse em comportamento transitório garantido e boa atenuação de distúrbios.

Este objetivo é formulado parcialmente por Tomei (1999) na forma de normas $L_{2}$ e $L_{\infty}$ arbitrariamente pequenas em relação aos distúrbios. Nesse trabalho, foi utilizada uma lei de controle combinando adaptação paramétrica e termos do tipo PD (Proporcional+Derivativo) com alto ganho, ajustados por um simples parâmetro $k$. As normas $L_{2}$ e $L_{\infty}$ resultam inversamente proporcionais ao ganho $k$, motivo pelo qual Tomei (1999), refere-se a desempenho transitório e a atenuação de distúrbios arbitrários. Entretanto, as seguintes observações devem ser consideradas:

a) A complexidade da lei de controle é significativamente maior do que a das leis existentes.

b) O principal inconveniente dessa lei de controle é que o domínio residual decresce às custas do aumento do ganho de um termo PD, amplificando o efeito de dinâmicas não modeladas e de ruídos de medição, além da amplificação do si- 
nal de controle, o qual pode crescer a ponto de provocar a saturação dos atuadores, colocando em risco a integridade e o desempenho do sistema.

c) O desempenho transitório devido a erros de rastreamento iniciais não é abordado.

Neste artigo, procuramos atingir os mesmos objetivos de uma maneira mais simples e eficiente, evitando os problemas de alto ganho de um termo PD. A idéia é a utilização de uma lei de controle em modo dual, originalmente referida por binária por Hsu e Costa (1994a). O artigo de Hsu et alii (1999), mais recente, generaliza e completa os resultados de Hsu e Costa (1994a). No artigo de Hsu e Aquino (1999a) essa lei de controle é aplicada no controle de manipuladores com rastreamento visual. Em Hsu et alii (1994), a lei de controle é combinada com uma função de saturação com o objetivo de atenuar o efeito indesejado provocado pela presença de distúrbios externos.

A adaptação em modo dual consiste essencialmente na utilização de um alto ganho de adaptação juntamente com uma projeção dos parâmetros estimados. Assim, para erros de rastreamento grandes, na fase transitória, o controlador age de modo próximo a um controlador deslizante, conseguindo-se convergência exponencial para um domínio residual arbitrariamente pequeno no espaço dos erros de rastreamento, e para erros menores, como uma lei de adaptação paramétrica. A lei de adaptação em modo dual possui diversas vantagens frente a outros algoritmos de adaptação ou de controle robusto conhecidos, entre elas a de apresentar sinais de controle contínuos, a de melhorar a robustez do sistema, e a de limitar o valor dos parâmetros estimados. A projeção tem o efeito de reduzir o ganho efetivo do controlador quando o erro de rastreamento aumenta. Deste modo, similarmente ao que ocorre com o controle deslizante, a sensibilidade a ruídos é menor.

O artigo está organizado da seguinte forma. A seção 2 apresenta o modelo matemático do manipulador. A lei de controle em modo dual é apresentada na seção 3. Segue-se a prova de estabilidade, de convergência e de atenuação de distúrbios na seção 4. Resultados de simulação são discutidos na seção 5 e as conclusões são apresentadas na seção 6 . Um apêndice complementa certas passagens matemáticas.

\section{MODELO MATEMÁTICO DO MANIPU- LADOR}

O modelo matemático de um manipulador com $n+1$ elos rígidos interconectados por $n$ juntas é dado por (Spong e
Vidyasagar, 1989, capítulo 6):

$$
\begin{array}{r}
\mathbf{D}(\mathbf{q}, \mathbf{a}) \ddot{\mathbf{q}}+\mathbf{C}(\mathbf{q}, \dot{\mathbf{q}}, \mathbf{a}) \dot{\mathbf{q}}+\mathbf{g}(\mathbf{q}, \mathbf{a})+\mathbf{f}(\mathbf{q}, \dot{\mathbf{q}}, \mathbf{a})= \\
\tau+\mathbf{d}(t)
\end{array}
$$

onde o vetor $\mathbf{q}=\left[q_{1} \ldots q_{n}\right]^{T}$ representa os deslocamentos relativos das juntas. $\mathrm{O}$ vetor $\mathbf{a} \in \Re^{m}$ representa $m$ parâmetros dependentes das constantes cinemáticas e dinâmicas do robô e dos atuadores, os quais consideraremos desconhecidos, a menos de valores nominais. Um fato importante é a dependência linear do modelo em relação aos elementos de a. $\mathrm{O}$ vetor $\tau$ denota as forças generalizadas aplicadas a cada junta; $\mathbf{D}(\mathbf{q}, \mathbf{a})$ é a matriz de inércia, simétrica e positiva definida; $\mathbf{C}(\mathbf{q}, \dot{\mathbf{q}}, \mathbf{a}) \dot{\mathbf{q}}$ representa as forças centrípetas e de Coriolis que afetam as juntas em movimento; $\mathbf{g}(\mathbf{q}, \mathbf{a})$ é o vetor de torque gravitacional; $\mathbf{f}(\mathbf{q}, \dot{\mathbf{q}}, \mathbf{a})$ é o vetor que representa os atritos associados a cada junta. A ação das forças perturbadoras externas são agrupadas no vetor $\mathbf{d}(t)$, o qual supomos de norma limitada uniformemente por uma constante.

$\mathrm{O}$ vetor a, embora desconhecido, supomos de um valor dentro de uma bola fechada de raio $\theta_{M}$ centrada no vetor $\mathbf{a}_{n o m}$ (o valor nominal de a), considerado conhecido. O desvio dos parâmetros em relação aos seus valores nominais é representado pelo vetor $\theta=\mathbf{a}-\mathbf{a}_{n o m}$. Estamos, portanto, supondo que o módulo deste vetor sempre será menor ou igual a $\theta_{M}$. Por simplicidade, mas sem perda de generalidade, consideraremos que os parâmetros incertos são constantes. A extensão ao caso variante no tempo é simples.

A escolha da matriz $\mathbf{C}(\mathbf{q}, \dot{\mathbf{q}}, \mathbf{a})$ não é única. Aqui definimos os elementos de $\mathbf{C}$ como em Spong e Vidyasagar (1989) e Spong (1995):

$$
c_{k j}=\sum_{i=1}^{n} \frac{1}{2}\left[\frac{\partial d_{k j}}{\partial q_{i}}+\frac{\partial d_{k i}}{\partial q_{j}}-\frac{\partial d_{i j}}{\partial q_{k}}\right] \dot{q}_{i}
$$

sendo $c_{k j}$ e $d_{k j}$ os elementos das matrizes $\mathbf{C e} \mathbf{D}$, respectivamente. No caso em que os parâmetros em a sejam constantes, é bem conhecido que, por um argumento de conservação da energia total, a matriz

$$
\frac{d \mathbf{D}(\mathbf{q}, \mathbf{a})}{d t}-2 \mathbf{C}(\mathbf{q}, \dot{\mathbf{q}}, \mathbf{a})
$$

é antisimétrica (Spong e Vidyasagar, 1989).

Esta propriedade é bem conhecida, sendo crucial na prova de estabilidade do algoritmo de controle adaptativo de Slotine e Li (1991). Porém, no caso variante no tempo, a igualdade acima não é válida e apenas a matriz seguinte é anti-simétrica (Tao, 1992):

$$
\sum_{i=1}^{n} \frac{\partial \mathbf{D}(\mathbf{q}, \mathbf{a})}{\partial q_{i}} \dot{q}_{i}-2 \mathbf{C}(\mathbf{q}, \dot{\mathbf{q}}, \mathbf{a})
$$




\section{ALGORITMO DE CONTROLE}

A trajetória desejada, denotada por $\mathbf{q}_{d}(t)$ é suposta uniformemente limitada, uniformemente contínua com as duas primeiras derivadas $\dot{\mathbf{q}}_{d}(t)$ e $\ddot{\mathbf{q}}_{d}(t)$ também uniformemente limitadas.

Seguindo a abordagem de Slotine e Li (1988), os seguintes erros são introduzidos:

$$
\tilde{\mathbf{q}}=\mathbf{q}-\mathbf{q}_{d} ; \quad \mathbf{s}=\dot{\tilde{\mathbf{q}}}+\lambda \tilde{\mathbf{q}} ; \quad \dot{\mathbf{q}}_{r}=\dot{\mathbf{q}}_{d}-\lambda \tilde{\mathbf{q}}
$$

sendo $\tilde{\mathbf{q}}(t)$ o erro instantâneo de rastreamento no espaço das juntas; $\mathbf{s}(t)$ é um erro auxiliar sendo $\lambda$ uma constante positiva. Note-se que cada elemento de $\tilde{\mathbf{q}}(t)$ pode ser visto como a saída de um filtro de primeira ordem com as componentes respectivas de $\mathbf{s}(t)$ como entrada. $\mathrm{O}$ vetor $\dot{\mathbf{q}}_{r}$ é um vetor auxiliar denominado velocidade de referência por Slotine e Li (1991) e Slotine e Li (1988).

$\mathrm{O}$ vetor $\hat{\mathbf{a}}=\mathbf{a}_{n o m}+\hat{\theta}$ representa uma estimativa adequada dos parâmetros; e $\tilde{\mathbf{a}}=\hat{\mathbf{a}}-\mathbf{a}=\hat{\theta}-\theta=\tilde{\theta}$, o erro de estimação, onde supomos que $\|\theta\| \leq \theta_{M}$, sendo $\theta_{M}$ um limite de incerteza suposto conhecido.

Note-se que, por considerarmos os parâmetros constantes, $\dot{\tilde{\mathbf{a}}}=\dot{\hat{\mathbf{a}}}=\dot{\hat{\theta}}=\dot{\tilde{\theta}}$.

Definimos a matriz de regressão conforme Slotine e Li (1991), i.e.,

$$
\begin{aligned}
& \mathbf{D}(\mathbf{q}, \mathbf{a}) \ddot{\mathbf{q}}_{r}+\mathbf{C}(\mathbf{q}, \dot{\mathbf{q}}, \mathbf{a}) \dot{\mathbf{q}}_{r}+\mathbf{g}(\mathbf{q}, \mathbf{a})+\mathbf{f}(\mathbf{q}, \dot{\mathbf{q}}, \mathbf{a})= \\
& \mathbf{Y}\left(\mathbf{q}, \dot{\mathbf{q}}, \dot{\mathbf{q}}_{r}, \ddot{\mathbf{q}}_{r}\right) \mathbf{a}
\end{aligned}
$$

onde fica explcitada a dependência linear do modelo dinâmico com as componentes do vetor $\mathbf{a}$.

A lei de controle proposta consiste na original de Slotine e Li (1988) (ver em Spong et alii (1990) uma análise detalhada) adicionando um terceiro termo robusto, sendo dada por:

$$
\tau=\mathbf{Y} \hat{\mathbf{a}}-\mathbf{K} \mathbf{s}-\bar{d} \mathcal{S} \mathbf{a t}(\alpha \mathbf{s})
$$

onde $\mathbf{K}$ é uma matriz simétrica positiva definida, $\bar{d}$ um ganho positivo, e $\alpha$ um escalar positivo. Chamamos $\mathcal{S}$ at $: \Re^{n} \rightarrow$ $\Re^{n}$ à função vetorial de saturação suave dada por:

$$
\mathcal{S a t}(\mathbf{y})=\frac{\mathbf{y}}{\|\mathbf{y}\|+1}
$$

A função de saturação $\mathcal{S}$ at é contínua com respeito a seu argumento, tem derivadas parciais contínuas, e suas componentes são limitadas ao intervalo $(-1,+1)$.

A Fig. 1 ilustra uma função de saturação suave de uma componente.

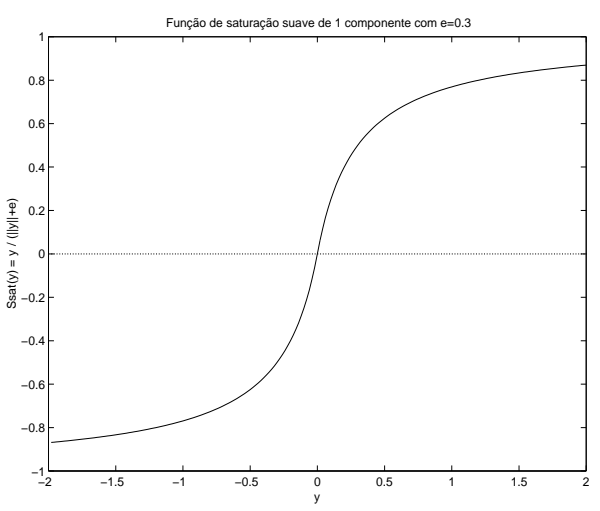

Figura 1. Função de saturação suave

A diferença desta lei com a lei adaptativa original de Slotine e Li (1988) reside na inclusão de projeção na lei de adaptação paramétrica, conforme detalhada na seção que segue, e no termo com a função de saturação que aqui se destina apenas a rejeitar perturbações ${ }^{1}$.

A equação dinâmica do sistema em malha fechada fica na forma (substituindo (7) em (1)):

$\mathbf{D}(\mathbf{q}, \mathbf{a}) \dot{\mathbf{s}}+\mathbf{C}(\mathbf{q}, \dot{\mathbf{q}}, \mathbf{a}) \mathbf{s}+\mathbf{K} \mathbf{s}=\mathbf{Y} \tilde{\mathbf{a}}+\mathbf{d}(t)-\bar{d} \mathcal{S} \mathbf{a t}(\alpha \mathbf{s})$

Para provar a estabilidade do algoritmo proposto escolhemos a seguinte candidata a função de Lyapunov:

$$
V(t)=\frac{1}{2}\left[\mathbf{s}^{T} \mathbf{D}(\mathbf{q}, \mathbf{a}) \mathbf{s}+\tilde{\mathbf{a}}^{T} \boldsymbol{\Gamma}^{-1} \tilde{\mathbf{a}}\right]
$$

sendo $\Gamma$ uma matriz simétrica positiva definida, a qual faz com que $V$ seja uma função escalar também positiva definida.

Derivando (10) com respeito ao tempo e considerando a antisimetria de (3) e a equação do sistema em malha fechada (9) chegamos a:

$$
\dot{V}=\mathbf{s}^{T}[\mathbf{Y} \tilde{\mathbf{a}}-\mathbf{K} \mathbf{s}+\mathbf{d}(t)-\bar{d} \mathcal{S} \mathbf{a t}(\alpha \mathbf{s})]+\tilde{\mathbf{a}}^{T} \boldsymbol{\Gamma}^{-1} \dot{\tilde{\mathbf{a}}}
$$

Em seguida, por considerarmos os parâmetros constantes, $\dot{\tilde{\mathbf{a}}}=\dot{\hat{\mathbf{a}}}=\dot{\tilde{\theta}}=\dot{\hat{\theta}}$, e considerando a definição (8) chegamos a:

$$
\dot{V}=-\mathbf{s}^{T} \mathbf{K} \mathbf{s}+\mathbf{s}^{T} \mathbf{d}(t)-\mathbf{s}^{T} \frac{\bar{d} \mathbf{s}}{\|\mathbf{s}\|+\varepsilon}+\tilde{\theta}^{T}\left[\mathbf{Y}^{T} \mathbf{s}+\boldsymbol{\Gamma}^{-\mathbf{1}} \dot{\hat{\theta}}\right]
$$

onde $\varepsilon:=1 / \alpha$.

Seguidamente, será analisada a lei de adaptação em modo dual a fim de continuar simplificando a igualdade anterior.

\footnotetext{
${ }^{1}$ em Slotine e Li (1988) tal função destinava-se a compensar o efeito de parâmetros não estimados exigindo uma função de saturação de amplitude modulada.
} 


\subsection{Lei de adaptação de modo dual}

A lei de adaptação proposta é:

$$
\dot{\hat{\theta}}=-\boldsymbol{\Gamma} \operatorname{Proj}\left(\mathbf{Y}^{\mathbf{T}} \mathbf{s}, \hat{\theta}\right) .
$$

onde $\operatorname{Proj}(\mathbf{y}, \hat{\theta})$ é o operador de projeção definido conforme $\operatorname{Proj}(\mathbf{y}, \hat{\theta})=$

$$
\left\{\begin{array}{cc}
\mathbf{y} & \text { se }\|\hat{\theta}\|<\theta_{M} \text { ou } \hat{\theta}^{T} \mathbf{y} \geq 0 \\
\left(\mathbf{I}-\frac{\hat{\theta} \hat{\theta}^{T}}{\|\hat{\theta}\|^{2}}\right) \mathbf{y} & \text { se }\|\hat{\theta}\| \geq \theta_{M} \text { e } \hat{\theta}^{T} \mathbf{y}<0
\end{array}\right.
$$

Este algoritmo de adaptação resulta nas seguintes propriedades (Hsu e Costa, 1994a):
(a) $\hat{\theta}(t)$ é uniformemente contínuo;
(b) se $\|\hat{\theta}(0)\| \leq \theta_{M}$, então $\|\hat{\theta}(t)\| \leq \theta_{M}, \forall t>0$;
(c) $\|\operatorname{Proj}(\mathbf{y}, \hat{\theta})\| \leq\|\mathbf{y}\|$;
(d) $\tilde{\theta}^{T} \operatorname{Proj}(\mathbf{y}, \hat{\theta}) \geq \tilde{\theta}^{T} \mathbf{y}$;
(e) $\|\operatorname{Proj}(\mathbf{y}, \hat{\theta})\|$ é limitado se $\|\mathbf{y}\|$ for também limitado.

A partir de (11) e da propriedade (d) do algoritmo de projeção, podemos afirmar que

$$
\begin{aligned}
& \dot{V} \leq-\mathbf{s}^{\mathbf{T}} \mathbf{K} \mathbf{s}+\mathbf{s}^{T} \mathbf{d}(t)-\mathbf{s}^{T} \bar{d} \frac{\mathbf{s}}{\|\mathbf{s}\|+\varepsilon} \\
& \dot{V} \leq-\mathbf{s}^{T} \mathbf{K} \mathbf{s}+\|\mathbf{s}\| d_{\max }-\bar{d} \frac{\|\mathbf{s}\|^{2}}{\|\mathbf{s}\|+\varepsilon}
\end{aligned}
$$

onde consideramos os distúrbios limitados por $\|\mathbf{d}(t)\| \leq$ $d_{\max }$.

Observe-se que não é possível afirmar que esta função seja negativa semidefinida. No que segue analisaremos as propriedades de estabilidade e de atenuação de distúrbios do sistema proposto.

\section{ESTABILIDADE E ATENUAÇÃO DE DISTÚRBIOS}

No caso do sistema não estar sujeito a distúrbios externos, i. é. $\mathbf{d}(t)=\mathbf{0}$, a equação (14) é dada por:

$$
\dot{V} \leq-\mathbf{s}^{T} \mathbf{K} \mathbf{s}-\bar{d} \frac{\|\mathbf{s}\|^{2}}{\|\mathbf{s}\|+\varepsilon} \leq 0
$$

o qual garante que $V(t)$ é monotonicamente decrescente e limitada superiormente, e portanto $\|\mathbf{s}\|$ é limitado superiormente ( $\|\tilde{a}\|$ é limitada também pelo operador de projeção no algoritmo de adaptação). Generalizando o teorema de LaSalle - Yoshizawa (ver Krstic et alii (1995, apêndice A)), de maneira similar a como é feito por Shevitz e Paden (1993), podemos afirmar que $\mathbf{s} \rightarrow \mathbf{0}$ quando $t \rightarrow \infty .^{2}$

É possível ainda demonstrar de modo similar a Hsu e Costa (1994a, apêndice B), que, se o ganho de adaptação for suficientemente alto e a matriz regressora $\mathbf{Y}$ calculada sobre a trajetória desejada for PE (persistently exciting), então o sistema completo de erros de estado e de erros paramétricos é globalmente exponencialmente estável. É interessante notar que o termo de saturação, inexistente em Hsu e Costa (1994a), não invalida o resultado.

Consideramos, em seguida, a presença de distúrbios externos. Neste caso, por conveniência, introduzimos a função que representa os dois últimos termos de (14):

$$
f(\|\mathbf{s}\|)=\|\mathbf{s}\| d_{\max }-\bar{d} \frac{\|\mathbf{s}\|^{2}}{\|\mathbf{s}\|+\varepsilon}
$$

Para $\bar{d} \leq d_{\max }: f(\|\mathbf{s}\|) \geq 0 \quad \forall\|\mathbf{s}\|$ e para $\bar{d}>$ $d_{\max }: f(\|\mathbf{s}\|)$ apresenta uma curva com regiões positivas e negativas. Mais precisamente $f(\|\mathbf{s}\|) \geq 0$ para $\|\mathbf{s}\| \in\left[0, d_{\max } \varepsilon /\left(\bar{d}-d_{\max }\right)\right]$, e $f(\|\mathbf{s}\|)<0$ para $\|\mathbf{s}\|>$ $d_{\max } \varepsilon /\left(\bar{d}-d_{\max }\right)$.

O intervalo do domínio no qual a função é positiva é tanto menor quanto maior for o ganho $\bar{d}$ e quanto menor for a constante $\varepsilon$, conseguindo-se que a função seja positiva só para uma região de valores de $\|\mathbf{s}\|$ arbitrariamente pequena. A título de ilustração, um exemplo é dado na Fig. 2, que mostra o gráfico da função $f(\|\mathbf{s}\|)$ para $d_{\max }=200, \bar{d}=205$, e $\varepsilon=0,5$.

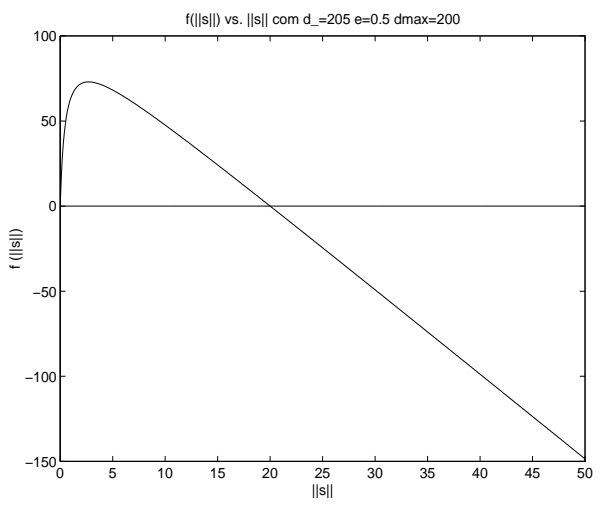

Figura 2. $f(\|\mathbf{s}\|)$ vs. $\|\mathbf{s}\|$

É possível conferir que o valor de $\|\mathbf{s}\|$ que maximiza a função $f(\|\mathbf{s}\|)$ é:

$$
\|\mathbf{s}\|_{M}=-\varepsilon+\varepsilon \sqrt{\frac{\bar{d}}{\bar{d}-d_{\max }}}>0
$$

\footnotetext{
${ }^{2}$ Para evitar complicações com sistemas descontí nuos, pode-se optar por utilizar uma projeção suave, como em Tomei (1999).
} 
sendo o valor máximo de $f(\|\mathbf{s}\|)$ dado por:

$$
\begin{aligned}
& f\left(\|\mathbf{s}\|_{M}\right)=\varepsilon d_{\max } \\
& {\left[\left(-1+\sqrt{\frac{\bar{d}}{\bar{d}-d_{\max }}}\right)-\frac{\bar{d}\left(-1+\sqrt{\frac{\bar{d}}{\bar{d}-d_{\max }}}\right)^{2}}{d_{\max } \sqrt{\frac{\bar{d}}{d-d_{\max }}}}\right]} \\
& =\varepsilon d_{\max } c(\bar{d})
\end{aligned}
$$

onde denotamos por $c(\bar{d})$ a expressão entre colchetes.

Seja $d_{0}$ um valor positivo qualquer, e escolhendo $\bar{d}$ como um parâmetro de projeto tal que $\bar{d} \geq d_{0}>d_{\max }$, a função $c(\bar{d})$ é sempre positiva no intervalo $\left[d_{0}, \infty\right)$, apresenta um máximo quando $\bar{d}=d_{o}$ e decresce tendendo para zero quando $\bar{d} \rightarrow$ $\infty$. Podemos, portanto, escrever:

$$
f(\|\mathbf{s}\|) \leq f_{\max }=\varepsilon d_{\max } c\left(d_{o}\right)
$$

onde $f_{\text {max }}$ pode ser reduzido a valores arbitrariamente pequenos diminuindo-se a constante $\varepsilon$ ou aumentando-se o parâmetro $d_{0}$ (ou, equivalentemente, aumentando $\bar{d}$ ). Utilizando a desigualdade acima em (14), obtemos:

$$
\dot{V} \leq-\mathbf{s}^{T} \mathbf{K} \mathbf{s}+\varepsilon d_{\max } c\left(d_{\mathrm{o}}\right)
$$

\subsection{Convergência exponencial dos erros}

Pela propriedade de não singularidade da matriz de inércia, podemos afirmar:

$$
C_{m}\|\mathbf{s}\|^{2} \leq \mathbf{s}^{T} \mathbf{D}(\mathbf{q}, \mathbf{a}) \mathbf{s} \leq C_{M}\|\mathbf{s}\|^{2}
$$

onde $C_{m}$ e $C_{M}$ são escalares positivos e constantes, se as juntas forem de revolução (Spong, 1995). Adotando, por comodidade, uma matriz $\mathbf{K}=k \mathbf{I}$, temos, de (19):

$$
\frac{C_{m}}{C_{M}} k\|\mathbf{s}\|^{2} \leq \frac{k}{C_{M}} \mathbf{s}^{\mathbf{T}} \mathbf{D}(\mathbf{q}, \mathbf{a}) \mathbf{s} \leq k\|\mathbf{s}\|^{2}=\mathbf{s}^{T} \mathbf{K} \mathbf{s}
$$

Substituindo em (18) e utilizando (10):

$$
\begin{aligned}
\dot{V} & \leq-\frac{k}{C_{M}} \mathbf{s}^{T} \mathbf{D}(\mathbf{q}, \mathbf{a}) \mathbf{s}+\varepsilon d_{\max } c\left(d_{\mathrm{o}}\right) \\
\dot{V} & \leq-\frac{2 k}{C_{M}} V+\frac{k}{C_{M}} \tilde{\mathbf{a}}^{T} \boldsymbol{\Gamma}^{-\mathbf{1}} \tilde{\mathbf{a}}+\varepsilon d_{\max } c\left(d_{\mathrm{o}}\right)
\end{aligned}
$$

Escolhendo, por comodidade, a matriz $\boldsymbol{\Gamma}=\gamma \mathbf{P}$, sendo $\gamma$ uma constante real positiva, e $\mathbf{P}$ uma matriz diagonal positiva definida, podemos escrever:

$$
\gamma^{-1} \frac{\|\tilde{\theta}\|^{2}}{\lambda_{M}(\mathbf{P})} \leq \tilde{\theta}^{T} \gamma^{-1} \mathbf{P}^{-\mathbf{1}} \tilde{\theta} \leq \gamma^{-1} \frac{\|\tilde{\theta}\|^{2}}{\lambda_{m}(\mathbf{P})}
$$

onde $\lambda_{m}(\mathbf{P})$ e $\lambda_{M}(\mathbf{P})$ denotam o mínimo e máximo autovalor da matriz P, respectivamente. Substituindo (21) em (20), e considerando que $\tilde{\mathbf{a}}=\tilde{\theta}$, obtemos:

$$
\dot{V} \leq-\frac{2 k}{C_{M}} V+\frac{k}{C_{M}} \gamma^{-1} \frac{\|\tilde{\theta}\|^{2}}{\lambda_{m}(\mathbf{P})}+\varepsilon d_{\max } c\left(d_{0}\right)
$$

Pela propriedade b) do algoritmo de projeção: $\|\hat{\theta}\| \leq \theta_{M} \Rightarrow$ $\|\tilde{\theta}\| \leq 2 \theta_{M}$. Assim, obtemos, da desigualdade anterior:

$$
\dot{V} \leq-\frac{2 k}{C_{M}} V+\frac{4 k \theta_{M}^{2}}{C_{M} \gamma \lambda_{m}(\mathbf{P})}+\varepsilon d_{\max } c\left(d_{0}\right)
$$

Introduzindo a constante $m=\frac{2 k}{C_{M}}$ e denotando por $R$ os dois últimos termos constantes da direita da equação (22), concluímos pelo lema de comparação:

$$
\begin{aligned}
V & \leq V(0) e^{-m t}+\frac{R}{m}\left(1-e^{-m t}\right) \Rightarrow \\
V & \leq V(0) e^{-m t}+\frac{2 \theta_{M}^{2}}{\gamma \lambda_{m}(\mathbf{P})}+\frac{\varepsilon d_{\max } c\left(d_{0}\right)}{m}
\end{aligned}
$$

Isto implica que a função $V$ é limitada superiormente, sendo portanto também o erro s limitado.

Pelas propriedades (19) e (21), podemos escrever

$$
V \geq \frac{1}{2}\left[C_{m}\|\mathbf{s}\|^{2}+\|\tilde{\theta}\|^{2} \frac{\gamma^{-1}}{\lambda_{M}(\mathbf{P})}\right] \geq \frac{C_{m}}{2}\|\mathbf{s}\|^{2}
$$

$\mathrm{e}$

$$
V(0) \leq \frac{1}{2}\left[C_{M}\|\mathbf{s}(0)\|^{2}+\frac{4 \theta_{M}^{2} \gamma^{-1}}{\lambda_{m}(\mathbf{P})}\right]
$$

Substituindo em (23) e rearranjando, obtemos:

$$
\begin{aligned}
\|\mathbf{s}\|^{2} \leq & {\left[\frac{C_{M}}{C_{m}}\|\mathbf{s}(0)\|^{2}+\frac{4 \theta_{M}^{2} \gamma^{-1}}{C_{m} \lambda_{m}(\mathbf{P})}\right] e^{-m t}+} \\
& \frac{4 \theta_{M}^{2} \gamma^{-1}}{C_{m} \lambda_{m}(\mathbf{P})}+\frac{2 \varepsilon d_{\max } c\left(d_{0}\right)}{C_{m} m}
\end{aligned}
$$

Substituindo $m$ pela sua expressão e considerando a desigualdade do triângulo aplicada à soma da última desigualdade, obtemos sucessivamente:

$$
\begin{aligned}
\|\mathbf{s}\| \leq & \sqrt{\frac{C_{M}}{C_{m}}}\|\mathbf{s}(0)\| e^{-\frac{m}{2} t}+\frac{2 \theta_{M} \gamma^{-\frac{1}{2}}}{\sqrt{C_{m} \lambda_{m}(\mathbf{P})}}\left(1+e^{-\frac{m}{2} t}\right) \\
& +\sqrt{\frac{C_{M}}{C_{m}} \frac{\varepsilon d_{\max } c\left(d_{0}\right)}{k}} \\
\|\mathbf{s}\| \leq & C\|\mathbf{s}(0)\| e^{-(m / 2) t}+k_{1} \gamma^{-1 / 2}+C \sqrt{\frac{\varepsilon d_{\max } c\left(d_{0}\right)}{k}}
\end{aligned}
$$

onde $C=\sqrt{C_{M} / C_{m}}$ e $k_{1}=4 \theta_{M} / \sqrt{C_{m} \lambda_{m}(\mathbf{P})}$. 
A desigualdade (24) implica que o erro auxiliar s tende exponencialmente a um resíduo arbitrariamente pequeno na medida em que se aumenta o ganho de adaptação $\gamma$, o parâmetro $d_{0}$ e/ou se diminui a constante $\varepsilon$. Observe-se que também poderia ser aumentado o ganho $k$ do termo PD (como Tomei (1999)) para reduzir este resíduo, mas isso acarretaria os inconvenientes já discutidos na Introdução, sendo, portanto, conveniente manter este ganho razoavelmente pequeno.

Como o erro de rastreamento $\tilde{\mathbf{q}}$ está relacionado ao erro auxiliar s por uma filtragem linear, a propriedade de convergência exponencial de $\mathbf{s}$ a um domínio residual é preservada para $\tilde{\mathbf{q}}$ e $\dot{\tilde{\mathbf{q}}}$, sendo possível verificar:

$$
\begin{aligned}
\mathbf{s} & =\dot{\tilde{\mathbf{q}}}+\lambda \tilde{\mathbf{q}} \Rightarrow \tilde{\mathbf{q}}=\mathbf{s} * e^{-\lambda t}+\tilde{\mathbf{q}}(0) e^{-\lambda t} \Rightarrow \\
\|\tilde{\mathbf{q}}\| & \leq\|\mathbf{s}\| * e^{-\lambda t}+\|\tilde{\mathbf{q}}(0)\| e^{-\lambda t}
\end{aligned}
$$

onde $*$ indica a convolução de sinais. Utilizando a desigualdade (24) obtemos:

$$
\begin{gathered}
\|\tilde{\mathbf{q}}\| \leq\|\tilde{\mathbf{q}}(0)\| e^{-\lambda t}+C\|\mathbf{s}(0)\|\left(e^{-(m / 2) t} * e^{-\lambda t}\right)+ \\
k_{1} \gamma^{-1 / 2} * e^{-\lambda t}+C \sqrt{\frac{\varepsilon d_{\max } c\left(d_{0}\right)}{k}} * e^{-\lambda t} \Rightarrow \\
\|\tilde{\mathbf{q}}\| \leq\|\tilde{\mathbf{q}}(0)\| e^{-\lambda t}+C \frac{\|\mathbf{s}(0)\|}{\lambda-m / 2}\left(e^{-(m / 2) t}-e^{-\lambda t}\right)+ \\
\quad \frac{k_{1}}{\lambda} \gamma^{-1 / 2}\left(1-e^{-\lambda t}\right)+\frac{C}{\lambda} \sqrt{\frac{\varepsilon d_{\max } c\left(d_{0}\right)}{k}}\left(1-e^{-\lambda t}\right) \\
\|\tilde{\mathbf{q}}\| \leq\|\tilde{\mathbf{q}}(0)\| e^{-\lambda t}+\frac{C}{|\lambda-m / 2|}\|\mathbf{s}(0)\| e^{-r t}+ \\
\quad \frac{k_{1}}{\lambda} \gamma^{-1 / 2}+\frac{C}{\lambda} \sqrt{\frac{\varepsilon d_{\max } c\left(d_{0}\right)}{k}}
\end{gathered}
$$

onde $r=\min (m / 2, \lambda)$. Resultado inteiramente análogo pode ser mostrado de maneira similar para $\|\dot{\tilde{\mathbf{q}}}\|$.

Para o caso em que os distúrbios estejam ausentes podemos verificar facilmente que as seguintes desigualdades são válidas:

$$
\begin{gathered}
\|\mathbf{s}\| \leq C\|\mathbf{s}(0)\| e^{-(m / 2) t}+k_{1} \gamma^{-1 / 2} \\
\|\tilde{\mathbf{q}}\| \leq\|\tilde{\mathbf{q}}(0)\| e^{-\lambda t}+\frac{C}{|\lambda-m / 2|}\|\mathbf{s}(0)\| e^{-r t}+\frac{k_{1}}{\lambda} \gamma^{-1 / 2}
\end{gathered}
$$

Desta forma demonstramos o seguinte teorema.

Teorema 1 A lei de controle adaptativo em modo dual (7) (12) aplicada ao manipulador modelado pela equação (1) sob a ação de distúrbios uniformemente limitados por $\|\mathbf{d}(t)\| \leq$ $d_{\max }$, com condição inicial dos parâmetros estimados satisfazendo $\|\hat{\theta}(0)\| \leq \theta_{M}$, e com $\bar{d} \geq d_{0}>d_{\max }$ resulta em limitação uniforme de todos os sinais do sistema em malha fechada, e, em particular, $\|\hat{\theta}(t)\| \leq \theta_{M}, \forall t \geq 0$. O erro de rastreamento $\tilde{\mathbf{q}}$ converge exponencialmente para um domínio residual dado por

$$
\|\tilde{\mathbf{q}}\| \leq O(1 / \sqrt{\gamma})+O\left(\varepsilon c\left(d_{0}\right) / k\right)
$$

No caso de ausência de distúrbios, a convergência exponencial ocorre com relação ao domínio residual

$$
\|\tilde{\mathbf{q}}\| \leq O(1 / \sqrt{\gamma})
$$

Neste caso temos ainda convergência assintótica do erro de rastreamento.

\section{SIMULAÇÕES}

Nesta seção serão apresentados os resultados de simulação obtidos com a lei de controle adaptativo em modo dual (7)(12) aplicada a um manipulador de duas juntas de revolução no mesmo plano. Supomos que o torque gravitacional e os atritos estáticos e dinâmicos são nulos, tal como é feito por Slotine e Li (1991, capítulo 9). Escolheremos para a simulação as seguintes constantes físicas com unidades em SI:

$m_{1}=1 ; l_{1}=1 ; m_{e}=2 ; \delta_{e}=30^{\circ} ; I_{1}=0,12 ; l_{c 1}=0,5$; $I_{e}=0,25 ; l_{c e}=0,6$

Introduzindo-se os seguintes parâmetros:

$a_{1}=I_{1}+m_{1} l_{c 1}^{2}+I_{e}+m_{e} l_{c e}^{2}+m_{e} l_{1}^{2} ; a_{2}=I_{e}+m_{e} l_{c e}^{2} ;$ $a_{3}=m_{e} l_{1} l_{c e} \cos \delta_{e} ; a_{4}=m_{e} l_{1} l_{c e} \sin \delta_{e}$;

o modelo dinâmico do manipulador é dado por:

$$
\begin{array}{r}
{\left[\begin{array}{ll}
d_{11} & d_{12} \\
d_{21} & d_{22}
\end{array}\right]\left[\begin{array}{l}
\ddot{q}_{1} \\
\ddot{q}_{2}
\end{array}\right]+\left[\begin{array}{cc}
-h \dot{q}_{2} & -h\left(\dot{q}_{1}+\dot{q}_{2}\right) \\
h \dot{q}_{1} & 0
\end{array}\right]\left[\begin{array}{c}
\dot{q}_{1} \\
\dot{q}_{2}
\end{array}\right]=} \\
{\left[\begin{array}{l}
\tau_{1} \\
\tau_{2}
\end{array}\right]+\left[\begin{array}{c}
d_{1} \\
d_{2}
\end{array}\right]}
\end{array}
$$

onde $d_{11}=a_{1}+2 a_{3} \cos q_{2}+2 a_{4} \sin q_{2} ; d_{12}=d_{21}=a_{2}+$ $a_{3} \cos q_{2}+a_{4} \sin q_{2} ; d_{22}=a_{2} ; h=a_{3} \sin q_{2}-a_{4} \cos q_{2}$.

As trajetórias desejadas $\left(\mathbf{q}_{d}\right)$ e suas duas primeiras derivadas ( $\dot{\mathbf{q}}_{d}$ e $\ddot{\mathbf{q}}_{d}$ ) são obtidas através da passagem de um sinal de referência $\mathbf{q}_{\text {ref }}$ por um filtro de segunda ordem criticamente amortecido, conforme $\ddot{\mathbf{q}}_{d}+\alpha \dot{\mathbf{q}}_{d}+\beta \mathbf{q}_{d}=\beta \mathbf{q}_{\text {ref }}$, onde foi escolhido $\alpha=20$ e $\beta=100$.

Esses sinais de referência são dados por

$$
\begin{aligned}
& q_{\text {ref } 1}=\frac{\pi}{4}+\frac{\pi}{2} \sin \left(\frac{2 \pi t}{2,5}\right)+\frac{\pi}{6} \sin (2 \pi t)+\frac{\pi}{10} \sin (4 \pi t) \\
& q_{\text {ref } 2}=\frac{\pi}{2}+\frac{\pi}{4} \sin \left(\frac{2 \pi t}{2,5}\right)+\frac{\pi}{8} \sin (4 \pi t)+\frac{\pi}{6} \sin (3 \pi t)
\end{aligned}
$$

Escolhemos os valores nominais dos parâmetros como: $a_{\text {nom } 1}=3,15 ; a_{\text {nom } 2}=0,85 ; a_{\text {nom } 3}=1,15 ; a_{\text {nom } 4}=$ 0,75 
de maneira tal que os erros reais dos parâmetros com respeito a seus valores nominais são:

$\theta_{1}=0,19 ; \theta_{2}=0,12 ; \theta_{3}=-0,11 ; \theta_{4}=-0,15$

As diversas simulações foram executadas com o programa Simnon, integrando pelo método de Euler com um passo de integração de $10^{-4}$ segundos.

Os ganhos do controlador, de adaptação, e a constante do filtro do erro $\mathbf{s}$ foram escolhidos conforme listados abaixo:

$\lambda=10 ; \boldsymbol{\Gamma}=\gamma \mathbf{P}$ onde $\mathbf{P}=\operatorname{diag}(0,07 ; 0,023 ; 0,02 ; 0,018)$ e $\gamma$ variável segundo a simulação; $\mathbf{K}=k \mathbf{I}$ onde $k=8$. O valor máximo do erro paramétrico $(\theta)$ é $\theta_{M}=0,35$.

As constantes da função de saturação suave $\bar{d}$ e $\varepsilon$ variaram com cada simulação também.

Os valores iniciais dos parâmetros estimados foram os valores nominais $\mathbf{a}_{n o m}$ (o qual implica que $\hat{\theta}(0)=0$ ). As duas juntas sofrem um distúrbio $d(t)=100 \mathrm{Nm} \sin (10 \pi t)$ para $0 \leq t<4 s$ e $d(t)=0$ para $t \geq 4 s$, o que implica que $d_{\max }=100 \mathrm{Nm}$.

Consideramos o manipulador inicialmente em repouso e na posição $\mathbf{q}(0)=\left[\begin{array}{ll}\pi / 4 & \pi / 4\end{array}\right]^{T}$.

\subsection{Simulações com distúrbios}
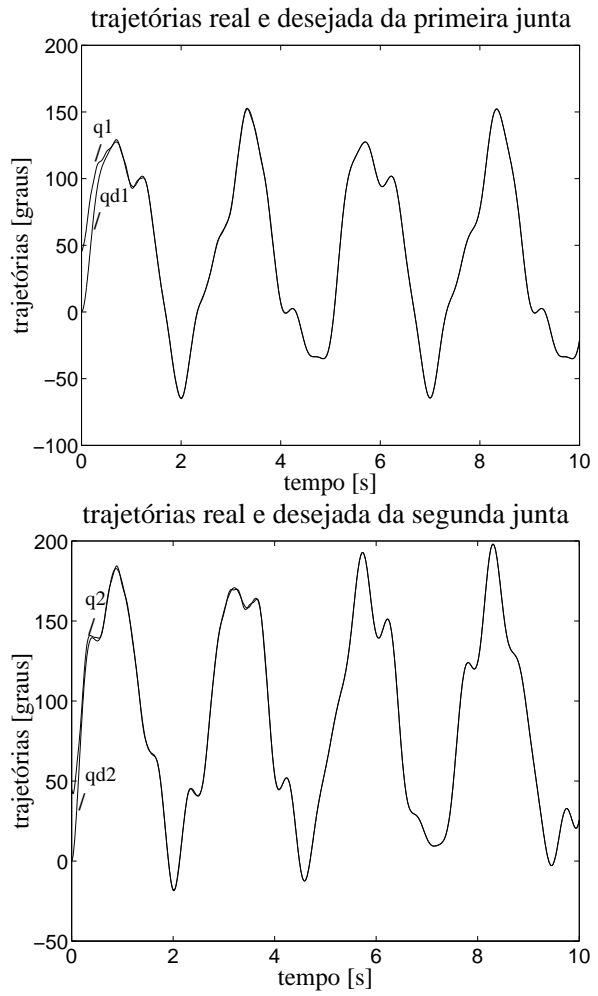
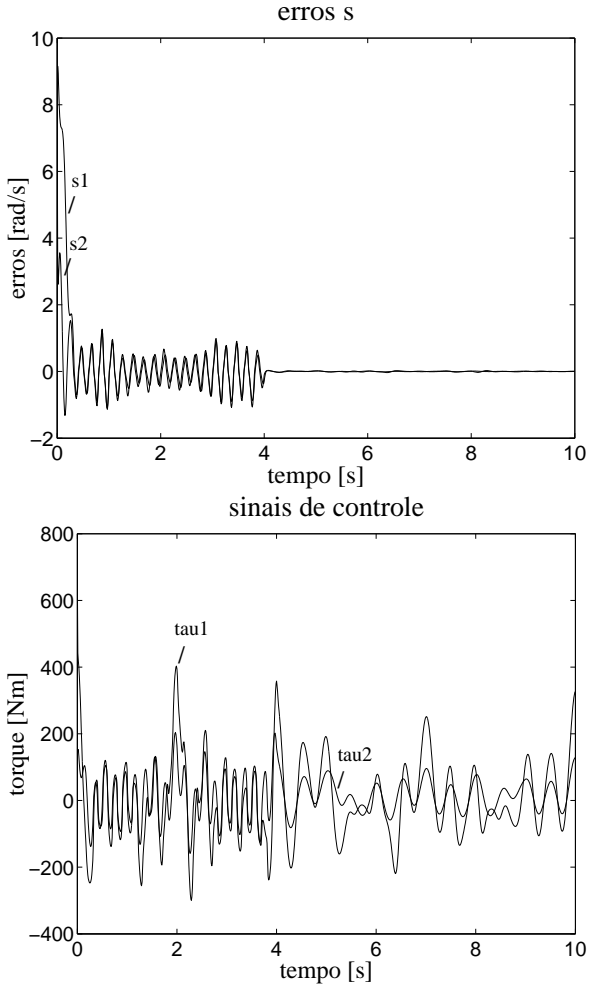

erro paramétrico

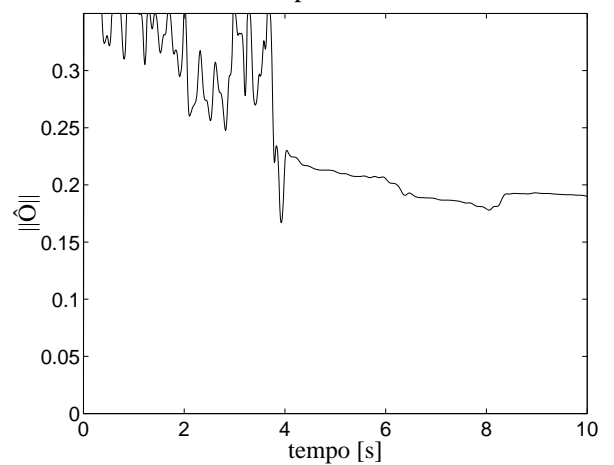

Figura 3. $\gamma=1,5 ; \bar{d}=125 ; \varepsilon=0,3$;

$$
\|\hat{O}\|=\|\hat{\theta}\| ; \tan _{i}=\tau_{i}
$$

Na primeira simulação, cujos resultados estão resumidos na Fig. 3, observamos como os erros de trajetória ficam limitados a um domínio residual, ao qual tendem rapidamente. Quando os distúrbios desaparecem ( $\mathrm{t}=4 \mathrm{~s}$.), os erros convergem rapidamente a zero. Nos gráficos das trajetórias de ambas as juntas esses erros são imperceptíveis após um período transitório razoavelmente rápido. No gráfico dos sinais de controle vemos que a presença dos distúrbios é responsável pelo intensa atividade inicial de controle, no sentido de rejeitar o distúrbio. No gráfico do módulo do vetor de parâmetros estimados, vemos que, depois de atingir inicialmente a fronteira da bola de projeção algumas vezes, tal vetor entra na bola permanecendo nela daí em diante. Conforme esperado, isto ocorre quando o erro de rastreamento é razoavelmente grande. Durante este período transitório, a ação de controle 
é similar à de um controle deslizante, obrigando o sistema a rapidamente convergir para a trajetória desejada.

\subsection{O efeito do parâmetro $\bar{d}$}
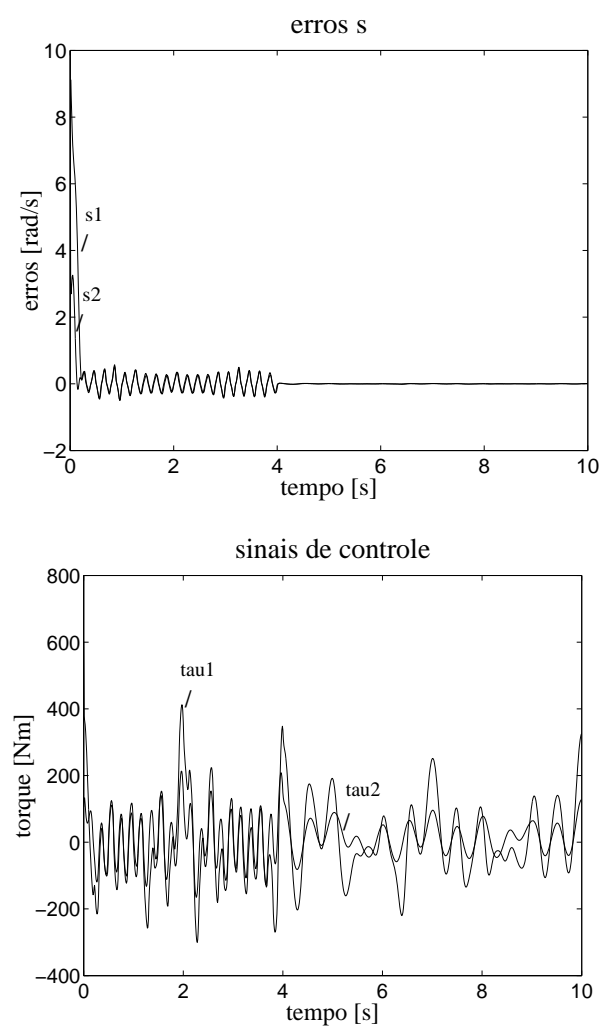

erro paramétrico

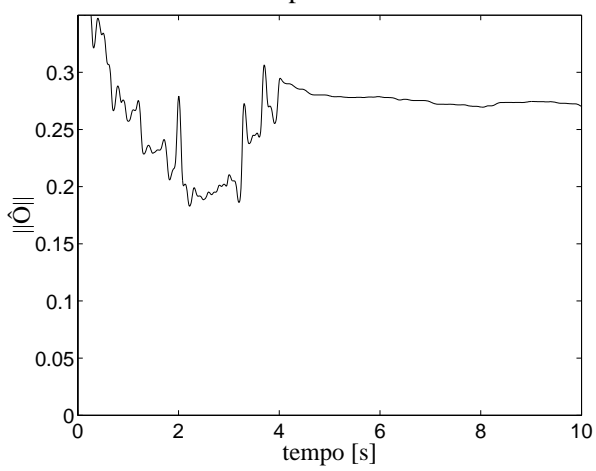

Figura 4. $\gamma=1,5 ; \bar{d}=200 ; \varepsilon=0,3$;

$$
\|\hat{O}\|=\|\hat{\theta}\| ; \operatorname{tau}_{i}=\tau_{i}
$$

Observamos na Fig. 4 o efeito de se aumentar a amplitude da função de saturação suave $\bar{d}$; a amplitude do resíduo para onde tendem os erros de rastreamento da trajetória diminuiu consideravelmente com respeito à simulação anterior sem afetar significativamente a amplitude do sinal de controle. Essa dimunuição nos erros de rastreamento faz com que os parâmetros estimados entrem rapidamente na bola de projeção, comportando-se o algoritmo de controle como uma lei de adaptação paramétrica dali em diante. Os gráficos das trajetórias reais e desejadas das juntas não foram incluídos por não apresentarem modificações sensíveis com respeito ao caso anterior.

\subsection{0 efeito de $\varepsilon$}
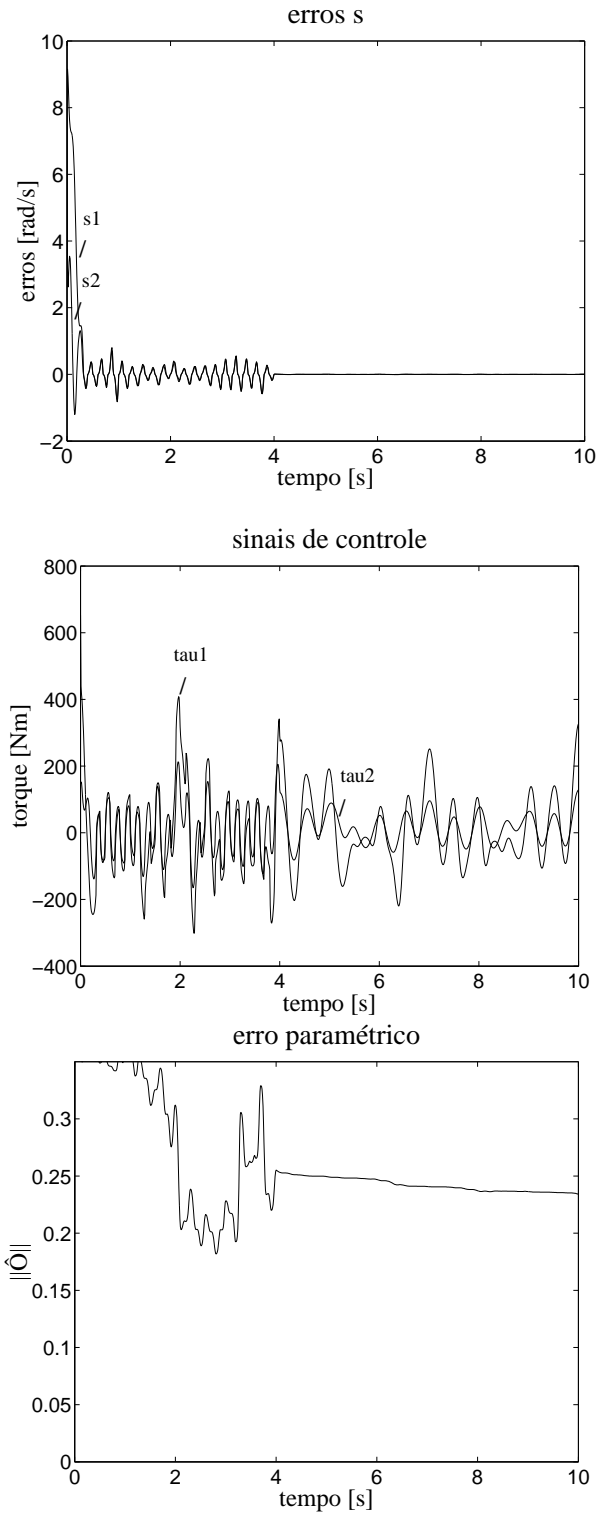

Figura 5. $\gamma=1,5 ; \bar{d}=125 ; \varepsilon=0,05$;

$$
\|\hat{O}\|=\|\hat{\theta}\| ; \operatorname{tau}_{i}=\tau_{i}
$$

Nesta simulação, cujos resultados são apresentados na Fig. 5, foi reduzida a "faixa linear"da função de saturação através da diminuição da constante $\varepsilon$. Dessa maneira, a ação de controle se aproxima mais à de um controle deslizante. $\mathrm{O}$ domínio residual ao qual tende o vetor do erro de rastreamento diminui também com respeito à primeira simulação. Assim, os parâmetros estimados entrem rapidamente na zona de adaptação paramétrica. 


\subsection{Simulações sem distúrbios e sem o termo robusto}
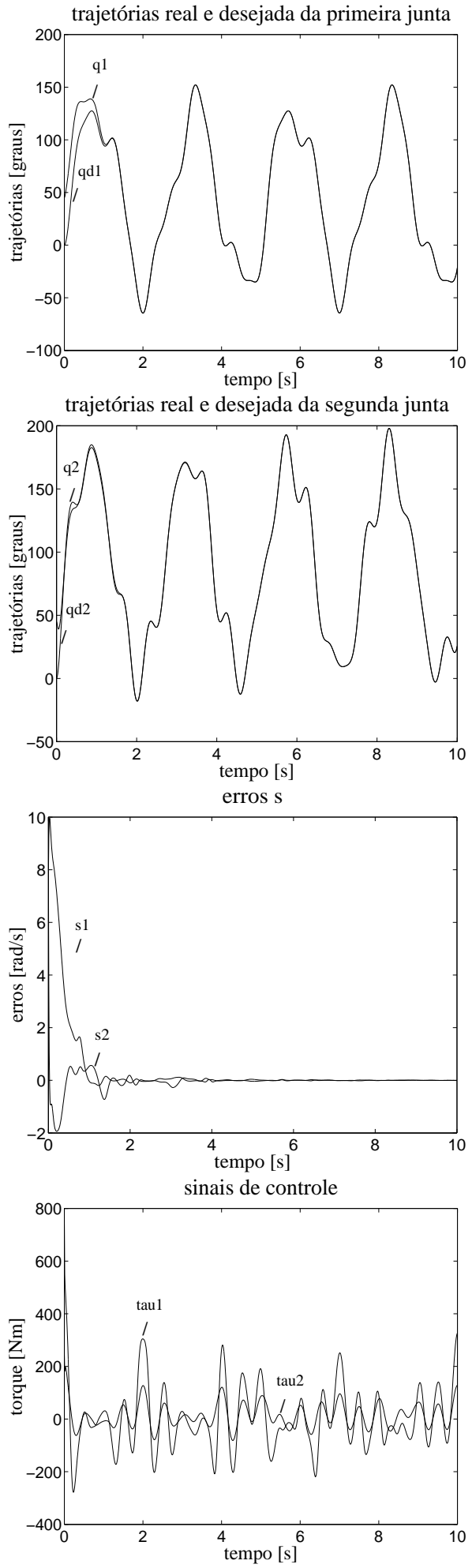

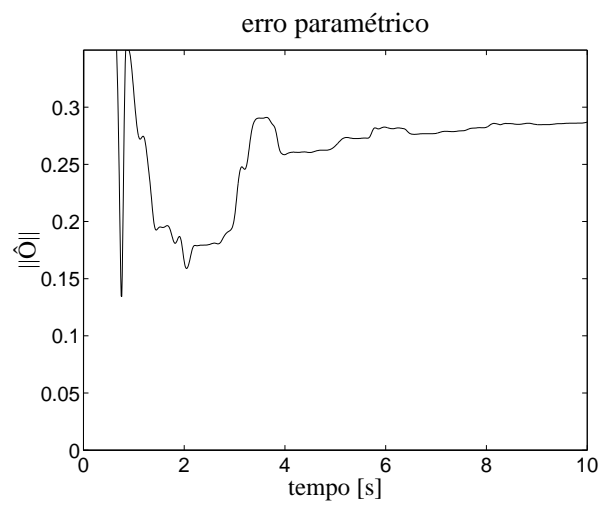

Figura $6 \gamma=1,5 ; \bar{d}=0 ;\|\hat{O}\|=\|\hat{\theta}\|$

$$
\operatorname{tau}_{i}=\tau_{i}
$$

Na Fig. 6 vemos o efeito de se "desligar"o termo robusto correspondente à saturação suave; o mecanismo de adaptação em modo dual é o único responsável por manter os erros dentro de valores limitados. Estes tendem a zero em forma mais lenta devido ao baixo valor do ganho PD. O sinal de controle é mais suave devido à ausência de distúrbios.

\subsection{Simulações sem o termo robusto e sem adaptação}
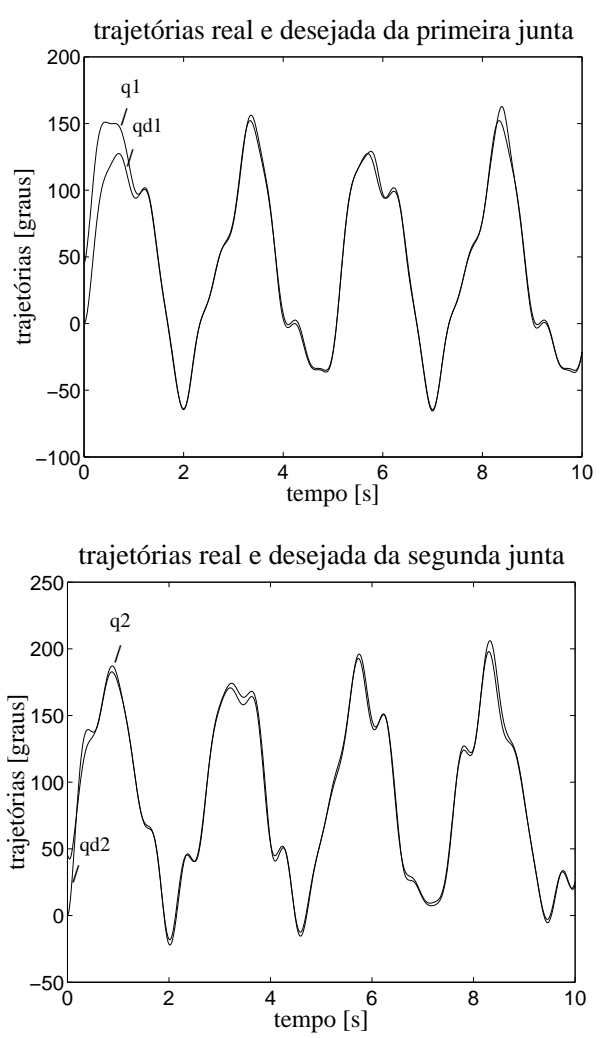

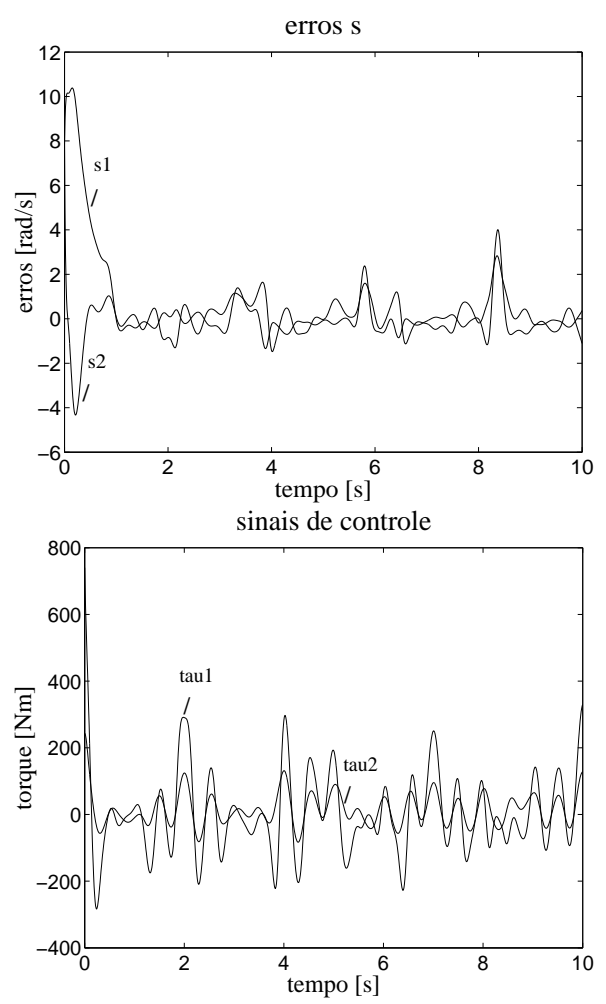

Figura $7 \gamma=0 ; \bar{d}=0$; $\operatorname{tau}_{i}=\tau_{i}$

Nesta simulação, com resultados mostrados na Fig. 7, foi desligada a adaptação e retirado o termo robusto. Isto significa que o controlador é limitado a um termo PD mais um termo não adaptativo ( $\hat{\mathbf{a}}=\mathbf{a}_{n o m}$ ). Vemos que não é comprometida a estabilidade do sistema, mas o desempenho piora consideravelmente, mostrando um alto erro de rastreamento de módulo não decrescente durante a totalidade do percurso simulado. Comparando com a simulação anterior, constatase a eficiência da adaptação em modo dual.

\section{CONCLUSÕES}

Apresentamos uma lei de adaptação em modo dual aplicada a robôs manipuladores. Utilizando a lei de controle adaptativo de Slotine e Li (1991), dotada de projeção paramétrica e alto ganho de adaptação, combinada com uma função de saturação suave, provamos que os erros de seguimento da trajetória tendem assintoticamente a zero e exponencialmente a um domínio residual de valor arbitrariamente pequeno. Além disso, diante da presença de distúrbios de entrada de amplitude limitada, o algoritmo mostrou-se capaz de atenuar seus efeitos, convergindo exponencialmente a um resíduo cuja amplitude depende dos ganhos da função de saturação suave, mas independe do ganho do termo PD do controlador. As simulações apresentadas evidenciam a redução dos efeitos dos distúrbios no rastreamento da trajetória desejada, e mostram que quando eles desaparecem, os erros convergem assintoticamente a zero.

\section{APÊNDICE A}

Prova da propriedade d) do algoritmo de projeção. Caso 1: $\|\hat{\theta}\|<\theta_{M}$ ou $\mathbf{y}^{T} \hat{\theta} \geq 0 \Rightarrow$

$$
\operatorname{Proj}(\mathbf{y}, \hat{\theta})=\mathbf{y} \quad \text { por definição. Portanto: }
$$

$$
\tilde{\theta}^{T} \operatorname{Proj}(\mathbf{y}, \hat{\theta})=\tilde{\theta}^{\mathbf{T}} \mathbf{y}
$$

Caso 2: $\|\hat{\theta}\| \geq \theta_{M}$ e $\mathbf{y}^{T} \hat{\theta}<0 \Rightarrow$

$$
\begin{aligned}
& \|\hat{\theta}\| \geq \theta_{M} \geq\|\theta\| \quad \text { por definição } \Rightarrow \\
& \|\hat{\theta}\| \geq\|\theta\| \Rightarrow \quad\|\hat{\theta}\|\left\|\mathbf{y}_{p}\right\| \geq\|\theta\|\left\|\mathbf{y}_{p}\right\| \quad \text { sendo } \\
& \mathbf{y}_{p}=\frac{\hat{\theta} \hat{\theta}^{T}}{\|\hat{\theta}\|^{2}} \mathbf{y} \text { a componente de } \mathbf{y} \text { sobre } \hat{\theta} \Rightarrow \quad-\hat{\theta}^{T} \mathbf{y}_{p} \geq \\
& \|\theta\|\left\|\mathbf{y}_{p}\right\| \quad \text { por hipótese } \hat{\theta}^{T} \mathbf{y}_{p}=\|\hat{\theta}\|\left\|\mathbf{y}_{p}\right\| \cos (\pi) \\
& \Rightarrow \quad \hat{\theta}^{T} \mathbf{y}_{p} \leq-\|\theta\|\left\|\mathbf{y}_{p}\right\| \leq \theta^{T} \mathbf{y}_{p} \\
& \Rightarrow \quad \tilde{\theta}^{T} \mathbf{y}_{p} \leq 0 \\
& \Rightarrow \quad-\tilde{\theta}^{T} \mathbf{y}_{p} \geq 0 \quad \Rightarrow \quad \tilde{\theta}^{T} \mathbf{y}-\tilde{\theta}^{T} \mathbf{y}_{p} \geq \tilde{\theta}^{T} \mathbf{y} \\
& \Rightarrow \quad \tilde{\theta}^{T}\left(\mathbf{y}-\mathbf{y}_{p}\right) \geq \tilde{\theta}^{T} \mathbf{y}
\end{aligned}
$$

e $\mathbf{y}-\mathbf{y}_{p}$ representa a componente perpendicular de $\mathbf{y}$ a $\hat{\theta}$ que é a projeção. Portanto

$$
\tilde{\theta}^{T} \operatorname{Proj}(\mathbf{y}, \hat{\theta}) \geq \tilde{\theta}^{T} \mathbf{y}
$$

\section{REFERÊNCIAS}

Bayard, D. e J. Wen (1988). New class of control laws for robotic manipulators. International journal of control, Vol. 47, $\mathrm{n}^{\circ}$ 5, pp 1387-1406.

Craig, J.J. (1986). Introduction to robotics, mechanics and control. Addinson - Wesley, Reading, MA.

Craig, J.J. (1988). Adaptive control of mechanical manipulators. Addinson - Wesley, Reading, MA.

Hsu, L., A. D. Araújo e R. Costa (1994). Analysis and design of I/O based variable structure adaptive control. IEEE transactions on automatic control (Jan.), Vol. 39, $\mathrm{n}^{\circ} 1$, pp 4-21.

Hsu, L. e R. Costa (1994a). B-MRAC: Global exponential stability with a new model reference adaptive controller based on binary control theory. Control theory and advanced technology (Dec.), Vol. 10, $\mathrm{n}^{\circ} 4$, part. 1, pp 649-668. 
Hsu, L., J. A. Real e R. Costa (1999). Dual mode adaptive control using gaussian networks: stability analysis. Proc. of the IFAC'99 World Congress, Beijing, China.

Hsu, L. e P. Aquino (1999a). Adaptive visual tracking with uncertain manipulator dynamic and uncalibrated camera. Proc. of the $39^{\text {th }}$ Conference on decision \& control $(C D C)$, Phoenix, USA.

Krstic, M., I. Kanellakopoulos e P. Kokotovic (1995). Nonlinear and adaptive control design. John Wiley \& Sons, New York.

Shevitz, D. e B. Paden (1993). Lyapunov stability theory of nonsmooth systems. Proc. of the $32^{\text {th }}$ conference on decision \& control $(C D C)$, San Antonio, Texas.

Slotine, J.J. e W. Li (1988). Adaptive manipulator control: A case study. IEEE transactions on automatic control (Nov.), Vol. 33, n 11, pp 995-1003.

Slotine, J.J. e W. Li (1991). Applied nonlinear control. Prentice - Hall, Englewood Cliffs, New Jersey.

Spong, M. e M. Vidyasagar (1989). Robot dynamic and control. Wiley, New York.

Spong, M., R. Ortega e R. Kelly (1990). Comments on 'Adaptive manipulator control: A case study'. IEEE transactions on automatic control (Jun.), Vol. 35, $\mathrm{n}^{\circ} 6$, pp 761-762.

Spong, M. (1995). Motion control of robot manipulators. Control handbook IEEE press.

Tao, G. (1992). On robust adaptive control of robots manipulators. Automatica, Vol. 28, pp 803-807.

Tomei, P. (1999). Robust adaptive control of robots with arbitrary transient performance and disturbance attenuation. IEEE transactions on automatic control (Mar.), Vol. 44, n 3, pp 654-658. 